\title{
ERRATUM
}

Elena K. Khlestkina · Ma Hla Myint Than

Elena G. Pestsova · Marion S. Röder

Sergey V. Malyshev · Viktor Korzun · Andreas Börner

\section{Mapping of 99 new microsatellite-derived loci in rye (Secale cereale L.) including 39 expressed sequence tags}

Published online: 12 February 2005

(C) Springer-Verlag 2005

\section{Theor Appl Genet (2004) 109:725-732}

In this paper, previously published results may have not been taken into account with sufficient clarity.

The detection of SSR fragments using tailed primers was originally reported by Oetting et al. (1995). The potential of rye ESTs for the development of new microsatellite markers has already been demonstrated by Hackauf and Wehling $(2002,2003)$. Using the same rye EST database, the authors developed 157 SCM markers and mapped 41 of these. Eighteen of the 39 REMS markers reported by us were derived from GenBank accessions already used for the development of SCM markers (Table 1). Thus, identical rye EST loci are probably represented by these eighteen REMS and SCM markers, respectively.

\section{References}

Hackauf B, Wehling P (2002) Identification of microsatellite polymorphisms in an expressed portion of the rye genome. Plant Breed 121:17-25

Hackauf B, Wehling P (2003) Development of microsatellite markers in rye: map construction. Plant Breed Seed Science 48:143-151

Oetting WS, Lee HK, Flanders DJ, Wiesner GL, Sellers TA, King RA (1995) Linkage analysis with multiplexed short tandem repeat polymorphisms using infrared fluorescence and M13 tailed primers. Genomics 30:450-458

The online version of the original article can be found at http:// dx.doi.org/10.1007/s00122-004-1659-z

E. K. Khlestkina · M. H. M. Than · E. G. Pestsova

M. S. Röder · S. V. Malyshev · V. Korzun · A. Börner $(\square)$

Institut für Pflanzengenetik und Kulturpflanzenforschung (IPK),

Corrensstrasse 3, 06466 Gatersleben, Germany

E-mail: boerner@ipk-gatersleben.de

E. K. Khlestkina

Institute of Cytology and Genetics,

Siberian Branch of the Russian Academy of Sciences,

Lavrentyeva ave. 10, Novosibirsk, 630090, Russia

M. H. M. Than

Department of Botany,

Mandalay University, Mandalay, Myanmar

E. G. Pestsova

Max-Planck-Institut für Züchtungsforschung,

Carl-von-Linnè-Weg 10, 50829 Cologne, Germany

S. V. Malyshev

Institute of Genetics and Cytology NASB,

220074 Minsk, Belarus

V. Korzun

Lochow-Petkus GmbH, PF 1197,

29296 Bergen, Germany 
Table 1 Survey of microsatellite markers (REMS) derived from rye EST database. REMS markers identical with SCMs are indicated

\begin{tabular}{|c|c|c|c|c|c|}
\hline Locus & SCM marker & $\begin{array}{l}\text { GenBank } \\
\text { accession no. }\end{array}$ & $\begin{array}{l}\text { Repeat type } \\
\text { and length }\end{array}$ & Expected size $(b p)^{3}$ & $\begin{array}{l}\text { Number } \\
\text { of alleles }\end{array}$ \\
\hline Xrems1280-1R & - & BF145382 & (CCT)6 & 199 & 2 \\
\hline Xrems1303-1R & - & BF146157 & (CTC) 5 & 309 & 2 \\
\hline Xrems1135-1R & - & BE493989 & (GA) 6 & 172 & 2 \\
\hline Xrems $1130-2 R$ & $\mathrm{SCM} 23^{1}$ & BE493797 & $(\mathrm{AAG}) 5$ & 232 & 2 \\
\hline Xrems1132-2R & SCM98 1 & BE493824 & $(\mathrm{GCT}) 5$ & 138 & 3 \\
\hline Xrems $1138-2 R$ & SCM $41^{1}$ & BE494083 & (CTC) 5 & 266 & 2 \\
\hline Xrems1194-2R & - & BE586335 & (TTC) 11 & 196 & 3 \\
\hline Xrems $1203-2 R$ & - & BE586786 & (GAA) 5 & 167 & 2 \\
\hline Xrems $1208-2 R$ & SCM $60^{1}$ & BE586891 & (CGC)6 & 143 & 2 \\
\hline Xrems $1208-2 R$ & 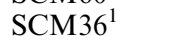 & BE588133 & $(\mathrm{AGC}) 6$ & 303 & 2 \\
\hline Xrems $1238-2 R$ & - & BE637241 & $(\mathrm{CGG}) 5$ & 286 & 2 \\
\hline Xrems $1251-2 R$ & SCM $188^{1}$ & BE704539 & (CATA) 5 & 261 & 2 \\
\hline Xrems $1254-3 R$ & SCM $170^{1}$ & BE704639 & $(\mathrm{CGG}) 5$ & 311 & 4 \\
\hline Xrems $1261-3 R$ & - & BE705070 & (GA) 6 & 252 & 5 \\
\hline Xrems $1323-3 R$ & $\mathrm{SCM} 154^{1}$ & BE704639 & (CT)7imp(TC)7 & 292 & 2 \\
\hline Xrems1135-3R & - & BE493989 & (GA)6 & 172 & 2 \\
\hline Xrems $1160-4 R$ & - & BE494651 & (TAG)7 & 228 & 3 \\
\hline Xrems1154-4R & - & BE494494 & (GCT) 8 & 134 & 3 \\
\hline Xrems1167-5R & SCM90 ${ }^{1}$ & BE494952 & $(\mathrm{CGG}) 5$ & 247 & 2 \\
\hline Xrems 1174-5R & - & BE495233 & $(\mathrm{GAGT}) 5$ & 302 & 4 \\
\hline Xrems 1186-5R & $\operatorname{SCM} 29^{1}$ & BE495963 & (CAC) 5 & 221 & 3 \\
\hline Xrems $1205-5 R$ & SCM $133^{1}$ & BE586813 & (ACAT)6 & 281 & 3 \\
\hline Xrems $1218-5 R$ & - & BE587316 & $(A G) 8$ & 230 & 3 \\
\hline Xrems $1237-5 R$ & - & BE637153 & (TAGC) 5 & 288 & 4 \\
\hline Xrems $1264-5 R$ & - & BE705252 & (CGTC) 5 & 282 & 2 \\
\hline Xrems 1266-5R & $\mathrm{SCM} 151^{2}$ & BE705296 & (GA) 8 & 202 & 3 \\
\hline Xrems $1152-6 R$ & SCM $55^{1}$ & BE494415 & (GCA) 5 & 251 & 3 \\
\hline Xrems 1247-6R & SCM $176^{2}$ & BE704499 & (TCC) 5 & 312 & 2 \\
\hline Xrems1250-6R & SCM168 1 & BE704532 & (GGC) 5 & 212 & 3 \\
\hline Xrems $1259-6 R$ & - & BE705058 & $(\mathrm{CGT}) 5$ & 271 & 4 \\
\hline Xrems 1154-6R & - & BE494494 & (GCT)8 & 134 & 2 \\
\hline Xrems1135-7R & - & BE493989 & (GA) 6 & 172 & 4 \\
\hline Xrems 1162-7R & SCM $3^{1}$ & BE494705 & $(\mathrm{GCC}) 5$ & 200 & 3 \\
\hline Xrems 1187-7R & - & BE496005 & (CAA) 5 & 215 & 2 \\
\hline Xrems $1188-7 R$ & $\mathrm{SCM} 19^{2}$ & BE496047 & (TC) 7 & 187 & 3 \\
\hline Xrems1197-7R & - & BE586481 & (CGC) 5 & 192 & 2 \\
\hline Xrems1234-7R & - & BE637039 & (AGC)6 & 256 & 2 \\
\hline Xrems1235-7R & $\mathrm{SCM} 183^{2}$ & BE704638 & (ATAG) 5 & 283 & 2 \\
\hline Xrems 1281-7R & - & BF 145397 & (CAA) 5 & 315 & 2 \\
\hline
\end{tabular}

${ }^{1}$ not mapped before

${ }^{2}$ mapped (Hackauf and Wehling 2002, 2003)
${ }^{3}$ with M13-extended primer

${ }^{4}$ detected in five parental rye genotypes 